\title{
Packing Changes in Melting, Freezing, and Coalescence of Titanium Nanoparticles from Atomic Simulations
}

\author{
LIN ZHANG ${ }^{1,2,3}$ and YAMING WANG ${ }^{2}$ \\ 1.-Key Laboratory for Anisotropy and Texture of Materials (Ministry of Education), Northeast- \\ ern University, Shenyang, China. 2.-Department of Materials Physics and Chemistry, School of \\ Materials Science and Engineering, Northeastern University, Shenyang, China. 3.-e-mail: \\ zhanglin@imp.neu.edu.cn
}

\begin{abstract}
Atomic simulations by using an embedded-atom-method potential were used to study changes of packing patterns in melting, freezing, and coalescence of titanium particles that contained tens to thousands of atoms. The packing evolution under dynamics processes leads to shape fluctuations. Small particles prefer icosahedron configurations. Large particles undergo hexagonal close-packed (HCP)-body-centered cubic (BCC) and BCC-melt transitions in a heating-cooling cycle. Calculations of specific heat are higher than those predicted from the classical Dulong-Petit law. Upon cooling, the hysteresis transition temperatures depend on the particle size and surface morphologies. The connection at room temperature results from contact and deformation between near facets of two particles. Complex facets exist after coalescence. A single-domain structure occurs for the coalescence of two relatively small particles. The occurrence accompanies the HCP-BCC transition, and the melting temperature is improved. In these large particles, coalescence particles consist of domains before melting.
\end{abstract}

\section{INTRODUCTION}

As a bridge between isolated atoms and bulk materials, metallic nanoparticles have attracted significant interest over several decades. ${ }^{1-8}$ Studies have shown that the most important chemical and physical properties of these particles are dependent on their geometrical structures and sizes. $^{9-11}$ Hexagonal structures have received significantly less attention than particles having bulk-phase structures of cubic crystals. Titanium (Ti) and titanium alloys exhibit a low density and adequate toughness, corrosion, wear, and fracture resistance. These make $\mathrm{Ti}$ an excellent choice in biomedical applications, such as orthopedic or dental implants. ${ }^{12-19}$ The biocompatibility of Ti alloys has been the subject of particular focus. In the most common medical-grade alloys, alloying elements such as aluminum and vanadium have been reported to be potentially harmful and toxic to the human body. ${ }^{20-25}$ This has created a need to improve mechanical properties of commercially pure (CP) $\mathrm{Ti}$ to a level comparable to other $\mathrm{Ti}$ alloys. Ti implants with porous structure would be an alternative clinical option in order to match the mechanical properties of bone and increase integration. In addition, the porous structure can offer space for bond regeneration without the aid of additional coating. However, conventional manufacturing technologies have great difficulties in processing the hexagonal $\alpha$-Ti with complex geometries. In addition, there is the structural transition to the $\mathrm{BCC} \beta$ - $\mathrm{Ti}$ at high temperatures. Additive manufacturing (AM) technology integrates mechanical, physical, chemical, biologic, and materialsscience engineering. In the AM processes, computer-aided design (CAD) software establishes the model and optimized topology of the products based on the three-dimensional (3D) data from computed tomography (CT) and magnetic resonance imaging (MRI). A computer controls movements of a local high-power heat source to melt individual or agglomerate $\mathrm{Ti}$ particles in large domains on a powder bed in a vacuum chamber, and let them solidify into one solid piece as the heat source moves away. After multiple melting-solidification cycles, this layer-by-layer fabrication can create near net parts with complex 3D morphology and internal 
microstructures with shorter lead times and raw material consumption. ${ }^{26-37}$ To date, nanoparticles have been used in the AM process. ${ }^{38,39}$ Their decrease in size results in different characteristics from their bulk counterparts and changes the atomic packing as well as yielding a temperature effect. The following interesting issues will naturally arise when considering the size dependence of Ti nanoparticles on their melting and freezing and coalescence involving combined behaviors of atomic packing changes and atomic movements. Because of lacking appropriate experimental techniques and the prohibitive computational expense of sophisticated ab initio approaches, molecular dynamics (MD) is a well-established technique to model various static and dynamic properties of metals reliably, in bulk or surface configurations. ${ }^{3,40-50}$

At the nanoscale, as the number of atoms in one particle increases, the computational cost increases exponentially, and the data complexity increases. In this MD work within the framework of the embedded-atom method (EAM), the validation of an atomic simulation by comparing the structural evolution and thermal behavior is of significant importance. Because bulk Ti has two structures, including $\mathrm{HCP}$ at low temperatures and $\mathrm{BCC}$ at high temperatures, ${ }^{51}$ there appears to be a dichotomy between high-temperature thermodynamic properties and low-temperature static configurations. A solution uses one potential for the BCC phase and the other for the HCP phase. By introducing an interpolating tanh function, a temperature-dependent Sommerfeld potential in the EAM form is produced as a linear combination of the two potentials. This potential preserves the low-temperature behavior up to room temperature and retains the high temperature behavior. ${ }^{52}$ However, the potential energy exhibits an apparent decrease in a temperature regime that corresponds with the HCP-BCC transition. The decrease results in a negative specific heat, but it should be positive. ${ }^{53}$ This work uses an EAM potential to describe the HCP-BCC and solid-liquid transitions, and microstructural simulations can be directly compared with the structural evolution and the heat capacity. The coalescence is of primary importance to understand the produced structures. Particles on a bed can come sufficiently close to agglomerating into larger particles. Thermodynamics indicates that under a focus heat, the shape evolution of coalescence particles has important consequences on the morphology-compact or ramified-depends, which indicates whether or not a smooth, uniform structure results. MD simulations provide the possibility of understanding processing-structureproperty-performance relations in the AM processes. The information from the simulations is helpful in producing desirable microstructural features and thus achieving comparable or even superior mechanical properties to conventionally manufactured parts.

\section{COMPUTATIONAL DETAILS}

To simulate particles containing more than hundreds of thousands of atoms, it is necessary to resort to an empirical description of the interatomic forces. We use the EAM interatomic potential for $\mathrm{Ti}$ derived by Pasianot and Savino. ${ }^{54,55}$ MD calculations were performed by using modified code from an old version of Moldy. The code uses a predictorcorrector algorithm to integrate equations of motion, and it provides the option to conduct extended-system simulations (for example, constant temperature and constant volume).

The initial structures of these particles were prepared as follows. "Spherical" particles were extracted from a $17.8 \mathrm{~nm} \times 25.7 \mathrm{~nm} \times 24.2 \mathrm{~nm}$ crystal cell of HCP Ti. Particles of 23 sizes were considered, from 13 to 3995 . They were labeled as $\mathrm{Ti}_{13}, \ldots$, and $\mathrm{Ti}_{3993}$, respectively, with diameters from $0.54 \mathrm{~nm}$ to $5.2 \mathrm{~nm}$. In MD simulations, if atoms are located near the boundary of the simulated cell under periodic boundary conditions, they should interact with other atoms in the imaging cells within the cutoff distance, which is determined from the EAM potential. To ensure the isolated state of the simulated atoms in a central position of the cell, the box size of the simulated central cell must be sufficiently large to avoid atomic interaction with the atoms of 26 neighboring cells. The $\mathrm{Ti}_{13}$ cluster, which is $<1 \mathrm{~nm}$, can be viewed as a typical magic cluster, and it constitutes an object of study in its own right. 56,57

After equilibration at room temperature, the particles were subjected to a heating-cooling cycle to identify structural transitions. Upon approaching the transition from either side, the temperature changed in steps of $50 \mathrm{~K}$. At each temperature, the system was first fully equilibrated in 900,000 time steps before running to accumulate statistics. A time step of $1.0 \times 10^{-15} \mathrm{~s}$ was used for all simulations. This fairly small value ensures stability of the trajectories during the runs to study atomic packing changes. The simulations were carried out in the NVT ensemble.

\section{RESULTS AND DISCUSSION}

Synchrotron radiation x-ray absorption spectroscopy (XAS) was applied to free titanium clusters/particles of $<1000$ atoms. These clusters/particles respectively include those $<55$ atoms, $80-150$ atoms, $300-500$ atoms, and $>600$ atoms. The absorption intensity evolves from a multi-peaked complex structure similar to that of $\mathrm{Ti}$ atoms toward spectra characterized by two main absorption features as in bulk titanium. ${ }^{58}$ We chose four particles that contained 13 atoms, 129 atoms, 305 atoms, and 611 atoms to exam their variations in potential energy per atom $E_{\mathrm{av}}$ with temperature. As one tensor, the moment of inertia can reflect the particle shape and mass distribution. Three values of principle axes were obtained by diagonalization of 
the tensor components. The shape factor was calculated from the minimum divided by the maximum principle value. Because rotation exists for one calculated particle, the increase in statistical time leads to a distortion of shape from average position coordinates, especially for small particles. Therefore, when calculating the shape factors of $\mathrm{Ti}_{13}$ and $\mathrm{Ti}_{129}$, we use an instantaneous position with a minimum energy in statistical time steps of the last 100,000 .

This approach is shown in Fig. 1 for four particles from $300 \mathrm{~K}$ to $1200 \mathrm{~K}$. The "as-made" HCP particles were first equilibrated at $300 \mathrm{~K}$ and then heated. After reaching $1600 \mathrm{~K}$, the particles were cooled. Because of the high ratio of surface atoms, the energy of the small particles exceeds that of the large particles. On heating and cooling, the energy of the $\mathrm{Ti}_{13}$ cluster keeps a stable value, whereas the energy changes of the other three particles present different behaviors. As plotted data on heating, the energy curve of $\mathrm{Ti}_{129}$ exhibits some characteristics indicating different packing in this particle. Followed a linear increase in 300-400 K, the energy decreases and then changes in a fluctuating way from $450 \mathrm{~K}$ to $750 \mathrm{~K}$. At $800 \mathrm{~K}$, there is an apparent increase. For $\mathrm{Ti}_{305}$, before a jump at $800 \mathrm{~K}$, the

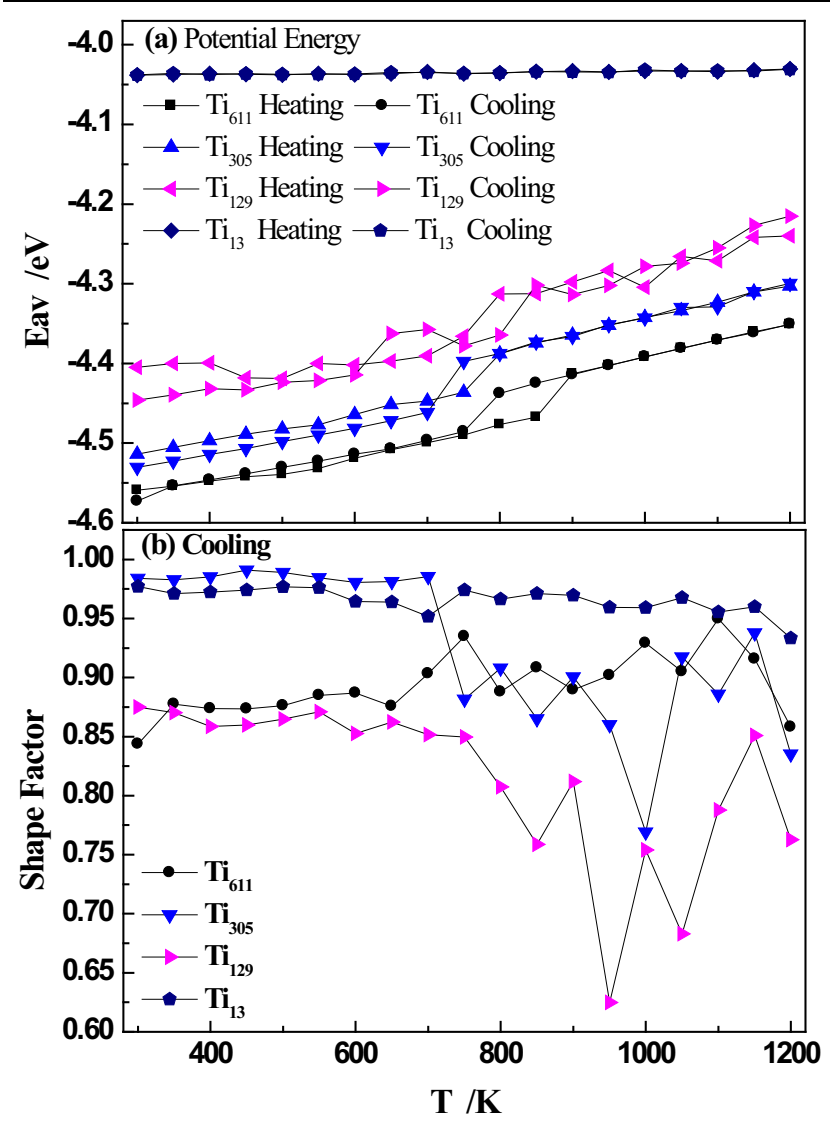

Fig. 1. Potential energy per atom and shape factor as a function of temperature for $\mathrm{Ti}_{13}, \mathrm{Ti}_{129}, \mathrm{Ti}_{305}$, and $\mathrm{Ti}_{611}$ particles. (a) Potential energy upon heating and cooling; (b) shape factor upon cooling. energy includes three increasing ranges separately in 300-550 K, 600-650 K, and 700-750 K. There are more temperature ranges before the atoms in $\mathrm{Ti}_{611}$ are packed in a disordered state, suggesting that there are continuous adjustments of the positions $<850 \mathrm{~K}$. On cooling, the energy still exhibits different characteristics for different particles. Therefore, in the following Figs. 2 and 3, we chose some typical packing images in different temperature ranges.

As shown the images, the $\mathrm{Ti}_{13}$ cluster has one stable icosahedral configuration, and its shape approximates that of a sphere. With heating $\mathrm{Ti}_{129}$ nanoparticle, the energy decreases, indicating that the icosahedral structure is more stable than the HCP packing for small particles having a free surface. The oscillating changes in data points are characteristics of atomic-packing changes in the icosahedral configurations. For the $\mathrm{Ti}_{305}$ or $\mathrm{Ti}_{611}$ particle, the melting transition is clearly identifiable from the abrupt increase in energy at $\mathrm{a} \sim 800$ $\mathrm{K}$ or $900 \mathrm{~K}$. Although atoms of $\mathrm{Ti}_{129}, \mathrm{Ti}_{305}$, and $\mathrm{Ti}_{611}$ particles still present HCP packing patterns at room temperature; as shown by the visual packing on heating, they differ from the initially constructed HCP particles. Because of finite time limitations in the MD simulations, surface atoms in these particles are packed with defects. As the temperature increases, the $\mathrm{Ti}_{305}$ and $\mathrm{Ti}_{611}$ particles exhibit a transition from HCP to BCC and BCC to melt. HCP and BCC local structures coexist in the $\mathrm{Ti}_{611}$ particle before finishing the HCP-BCC transition. Then, this particle exhibits a rearrangement of some surface atoms prior to melting. The structural rearrangements in the surface occur in a temperature range, and the disordered region spreads rapidly through the particle at a high temperature. These phenomena also occur in other particles with larger diameters. Upon cooling, the energy of $\mathrm{Ti}_{129}$ decreases, and then only a few atoms adjust their positions in one relatively stable configuration. The $\mathrm{Ti}_{305}$ or $\mathrm{Ti}_{611}$ undergoes a sharp liquid-solid transition despite a strong hysteresis. After freezing, $\mathrm{Ti}_{305}$ does not present an apparent structural transition. However, the energy point indicates that at $300 \mathrm{~K}$ the packing pattern of $\mathrm{Ti}_{611}$ differs from that above room temperature. Figure $1 b$ shows the shape factors of these four particles upon cooling. The shape factor of the $\mathrm{Ti}_{13}$ cluster closes to 1.0, indicating its near-spherical shape. For the other three particles, there are significant shape fluctuations before crystallization. Then, their shapes keep relative stability after freezing, where the $\mathrm{Ti}_{305}$ particle has a near-spherical shape. Except for the $\mathrm{Ti}_{611}$ particle, the other three particles present icosahedral configurations upon cooling, as shown in Fig. 3.

Only $\mathrm{Ti}_{611}$ has transitions, including $\mathrm{HCP}$ to $\mathrm{BCC}$, solid-liquid/liquid-solid, and BCC to HCP during melting/freezing, which can be identified with transition temperatures. Therefore, we will only discuss 


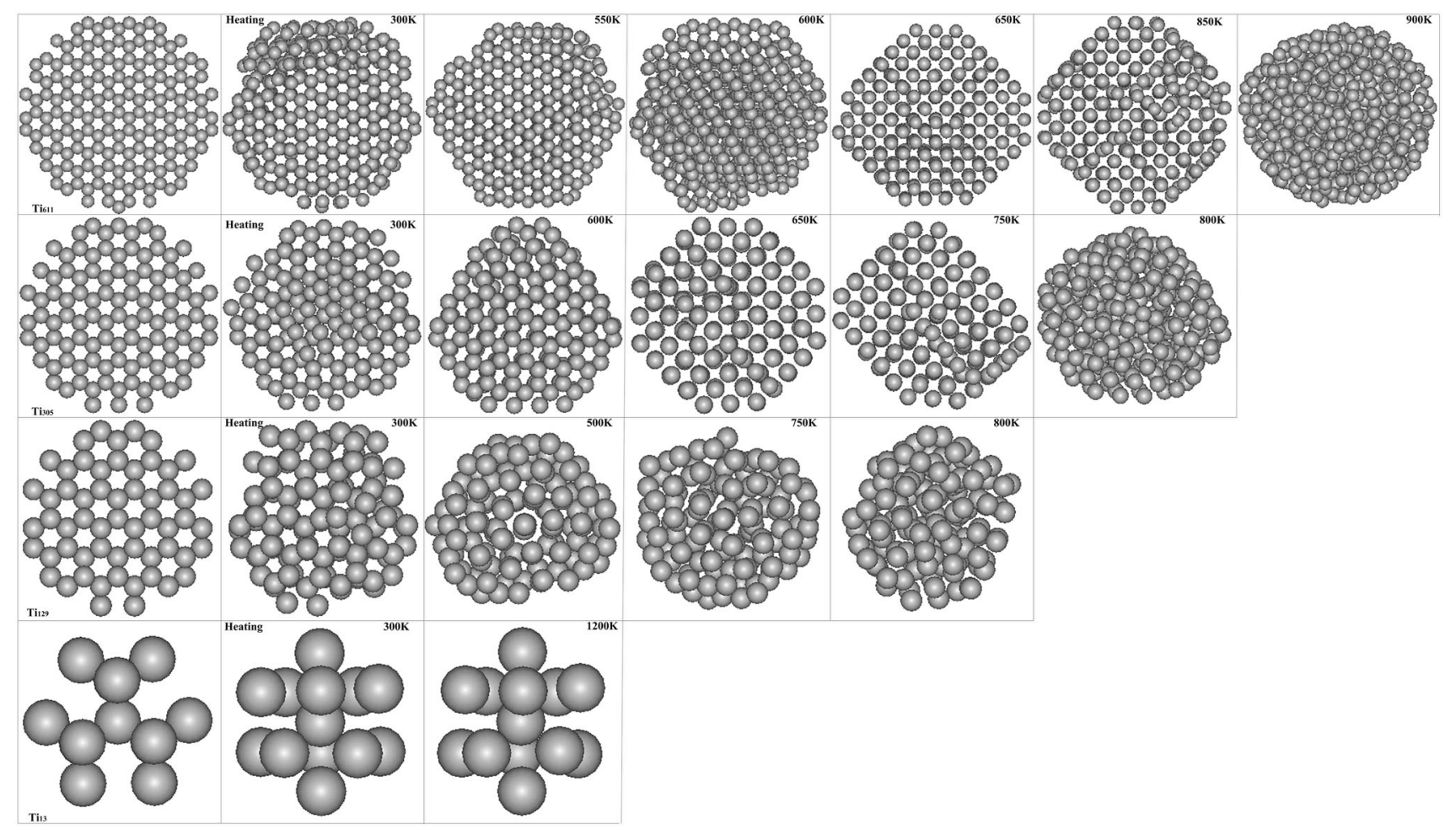

Fig. 2. Atomic packing of $\mathrm{Ti}_{13}, \mathrm{Ti}_{129}, \mathrm{Ti}_{305}$, and $\mathrm{Ti}_{611}$ particles upon heating.

particles that contain $>600$ atoms. The search for melting, freezing, and packing transition temperatures is performed by a "bisection" algorithm to narrow the search range until the temperatures are found. In these large particles, a considerable proportion of atoms occurs at the surface. Their movements and rearrangements have a large influence on the transitions. Figure 4 shows that the melting temperature versus size increases rapidly and then increases slowly with an increase in particle size. The increases agree reasonably well with predictions based on macroscopic concepts. The freezing temperature shows only small oscillations with an increase in diameter, and the increase in hysteresis temperature indicates a large undercooling temperature for the freezing of one large particle. In the cases of $\mathrm{HCP}-\mathrm{BCC}$ and $\mathrm{BCC}-\mathrm{HCP}$ transitions, the transition temperatures present oscillating changes as the diameter increases. Table I lists these melting, freezing, HCP-BCC, and BCC-HCP transition temperatures for these particles.

As the energy changes with increasing temperature, the thermal capacity can be determined by $\Delta U /$ $\Delta T$, where $\Delta U$ is composed of the potential energy and kinetic energy. In the crystals, the atoms at lattice points present thermal movements around the points. The Dulong Petit law indicates that the increase in slope is three for the total energy varying with the temperature, ${ }^{59}$ where half originates from the kinetic-energy contribution and the other half is from the potential energy. Compared with atoms in the inner region of the particles, surface and near-surface atoms have fewer coordination atoms. These atoms only need a small amount of energy to change their positions. Figure 5 shows that, because of the stable icosahedral configuration of $\mathrm{Ti}_{13}$, the slope is zero. For the small particles, a decrease in the proportion of the surface atoms results in a rapid increase in the slopes of these particles. As the proportion of surface atoms is reduced, most of the atoms in these large particles can hold their HCP packing patterns in a larger temperature range. Because the $\mathrm{HCP}-\mathrm{BCC}$ transition requires more energy, the slope value is $>1.5$ for nanoparticles with diameters $>3 \mathrm{~nm}$.

To understand the packing evolution of coalesced particles upon heating, the evolution is considered for standing free particles with the same size. The melting temperature of the coalescence particle differs from that of the original single particle. In the coalescence simulations, two HCP particles at $300 \mathrm{~K}$ were used as the starting-point configuration. Here, they were placed in contact with their $[\overline{1} 2 \overline{1} 0]$ facets, i.e., at a distance of $\sim 0.3 \mathrm{~nm}$, which is roughly equal to the nearest-neighbor distance in bulk Ti. To identify the atoms separately in the two particles, we use bi-colored images to show the atom packing in Fig. 6. 


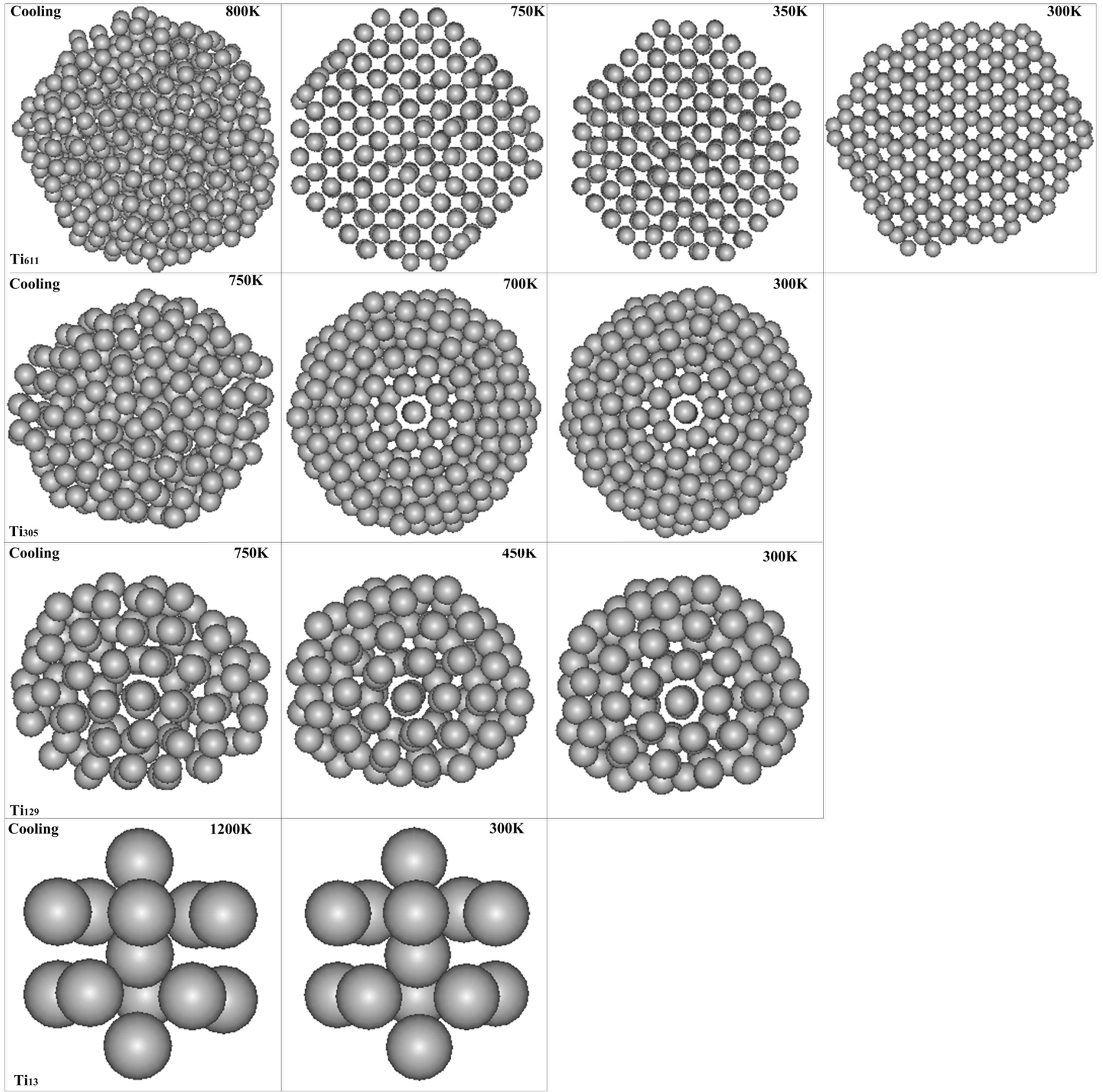

Fig. 3. Atomic packing of $\mathrm{Ti}_{13}, \mathrm{Ti}_{129}, \mathrm{Ti}_{305}$, and $\mathrm{Ti}_{611}$ particles upon cooling.

Figure 7 shows that the coalescence particles at $300 \mathrm{~K}$ are elongated, and a connection region is formed. Upon heating, some surface atoms obtain extra energy and move toward the connection region. For the relatively small $\mathrm{Ti}_{611}$ particles in three pairs, the coalesced particle presents apparent packing changes compared with those of the larger particles. Above $600 \mathrm{~K}$, an $\mathrm{HCP}-\mathrm{BCC}$ transition occurs for the three pairs, and shape changes present differences. Small shape factors indicate that these particles have complex curvatures and more edges in the surface, which limit the surfaceatom movements necessary for particle spheroidization. For the two $\mathrm{Ti}_{611}$ particles, the coalesced particle is more spherical than the other two pairs $<900 \mathrm{~K}$. At $1000 \mathrm{~K}$, the spherical shape indicates melting of the coalescence particle. The melting temperature of a single $\mathrm{Ti}_{611}$ is $880 \mathrm{~K}$. Therefore, the melting temperature increases imply that one uniform particle is produced after the 


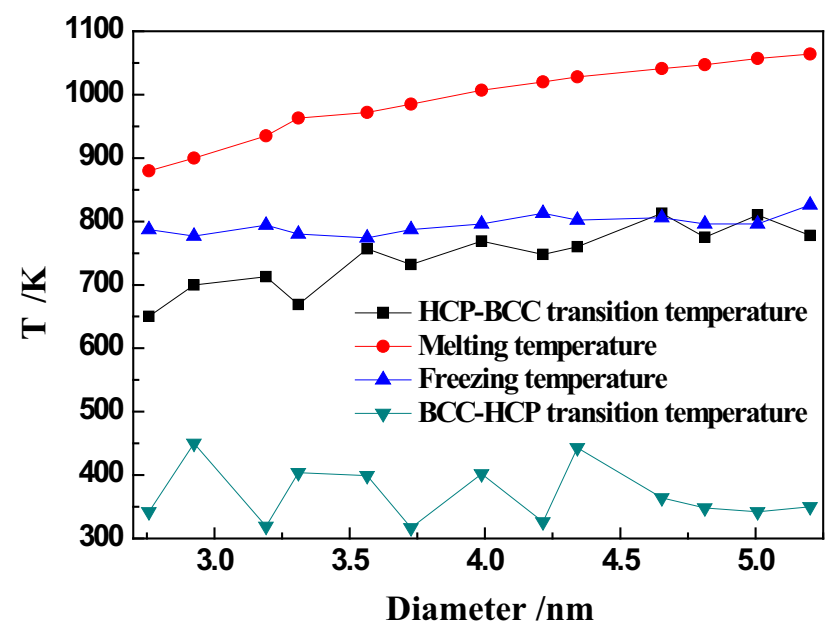

Fig. 4. Melting, freezing, and $\mathrm{HCP}-\mathrm{BCC}$ transition temperatures for various particles.

Table I. Melting, freezing, HCP-BCC, and BCCHCP transition temperatures of the particles with diameters $>2.76 \mathrm{~nm}$

\begin{tabular}{|c|c|c|c|c|}
\hline \multirow[t]{2}{*}{$D(\mathbf{n m})$} & \multicolumn{4}{|c|}{$T(\mathbf{K})$} \\
\hline & $T_{\text {Melting }}$ & $T_{\text {Freezing }}$ & $T_{\text {HCP-BCC }}$ & $T_{\text {BCC-HCP }}$ \\
\hline $2.76\left(\mathrm{Ti}_{611}\right)$ & 880 & 787 & 650 & 342 \\
\hline $2.92\left(\mathrm{Ti}_{763}\right)$ & 900 & 777 & 700 & 450 \\
\hline $3.20\left(\mathrm{Ti}_{919}\right)$ & 934 & 794 & 713 & 319 \\
\hline $3.31\left(\mathrm{Ti}_{1111}\right)$ & 963 & 780 & 669 & 404 \\
\hline $3.56\left(\mathrm{Ti}_{1285}\right)$ & 972 & 774 & 757 & 399 \\
\hline $3.73\left(\mathrm{Ti}_{1555}\right)$ & 985 & 787 & 731 & 317 \\
\hline $3.99\left(\mathrm{Ti}_{1809}\right)$ & 1007 & 796 & 769 & 402 \\
\hline $4.21\left(\mathrm{Ti}_{2115}\right)$ & 1020 & 813 & 748 & 326 \\
\hline $4.34\left(\mathrm{Ti}_{2403}\right)$ & 1028 & 802 & 760 & 443 \\
\hline $4.65\left(\mathrm{Ti}_{2763}\right)$ & 1041 & 806 & 813 & 364 \\
\hline $4.81\left(\mathrm{Ti}_{3129}\right)$ & 1047 & 796 & 775 & 348 \\
\hline $5.01\left(\mathrm{Ti}_{3545}\right)$ & 1056 & 796 & 810 & 342 \\
\hline $5.20\left(\mathrm{Ti}_{3995}\right)$ & 1064 & 826 & 778 & 350 \\
\hline
\end{tabular}

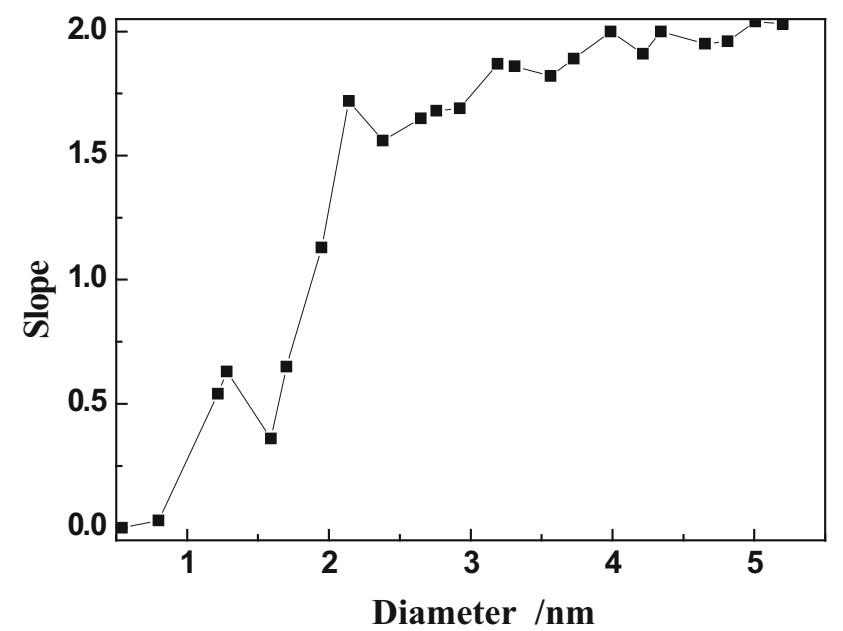

Fig. 5. Heating capacity slope of various particles.
HCP-BCC transition. For large particles, the melting points of the coalesced particles do not present an apparent increase compared with the single particle. The atom packing from Fig. 6 shows that at $300 \mathrm{~K}$, a connection region forms, owing to the compressed deformation of the two $\mathrm{Ti}_{611}$ or $\mathrm{Ti}_{919}$ particles and small stretching results for the $\mathrm{Ti}_{1809}$ particles. In each of the three pairs, a relative slip exists between two particles. At $800 \mathrm{~K}$, the two $\mathrm{Ti}_{611}$ particles are combined into one $\mathrm{Ti}_{1222}$ particle with BCC packing patterns. In the case of the two $\mathrm{Ti}_{919}$ particles, although no apparent interface exists between them at $800 \mathrm{~K}$, the shape indicates that the two particles have not been combined into one unity. For two $\mathrm{Ti}_{1809}$ particles, at $800 \mathrm{~K}$, although the atom packing in every particle has been transformed into BCC packing, an interface region still exists between them. An increase in temperature changes the particle shape, and "spherical" particles occur in melt states.

\section{CONCLUSION}

Atomic simulations of some dynamic and thermodynamic properties of unsupported Ti nanoparticles were used to study packing transitions in melting, freezing, and coalescence. During melting small particles having $<300$ atoms present a transition from HCP packing to icosahedral configurations. The HCP-BCC transition can be found in these particles that contain $>300$ atoms. For these particles containing $>600$ atoms, their melting behaviors present similarities including that surface atoms rearrange in a temperature range after the HCP-BCC transition, their melting temperatures are identifiable, and melting rapidly proceeds from the surface inwards. Evidence exists for a slope that corresponds to the heat capacity, which are on the applicability of macroscopic concepts for bulk materials. The simulation finding addresses that, owing to the HCP-BCC transition, the slope from the contribution of the potential is close to 2.0. During freezing these large particles containing $>600$ atoms undergo liquid-BCC and BCC-HCP transitions and require a large undercooling temperature for the liquid-solid transition. These small particles prefer an icosahedral configuration after freezing. After contact and deformation, a connection region forms between two particles. Accompanied by atom movements toward the region, it increases. Owing to the nanocrystal facets, the coalescence particles are not "spherical." For the relatively small particles, as the HCP-BCC transition occurs, a singledomain structure in the coalescence particle results. For the larger particles, although atoms in the coalesced particle are packed in the BCC structures, complex internal structures occur. Thus, high-energy extended defects prevent crystallization into a single domain. 
Packing Changes in Melting, Freezing, and Coalescence of Titanium Nanoparticles from Atomic Simulations

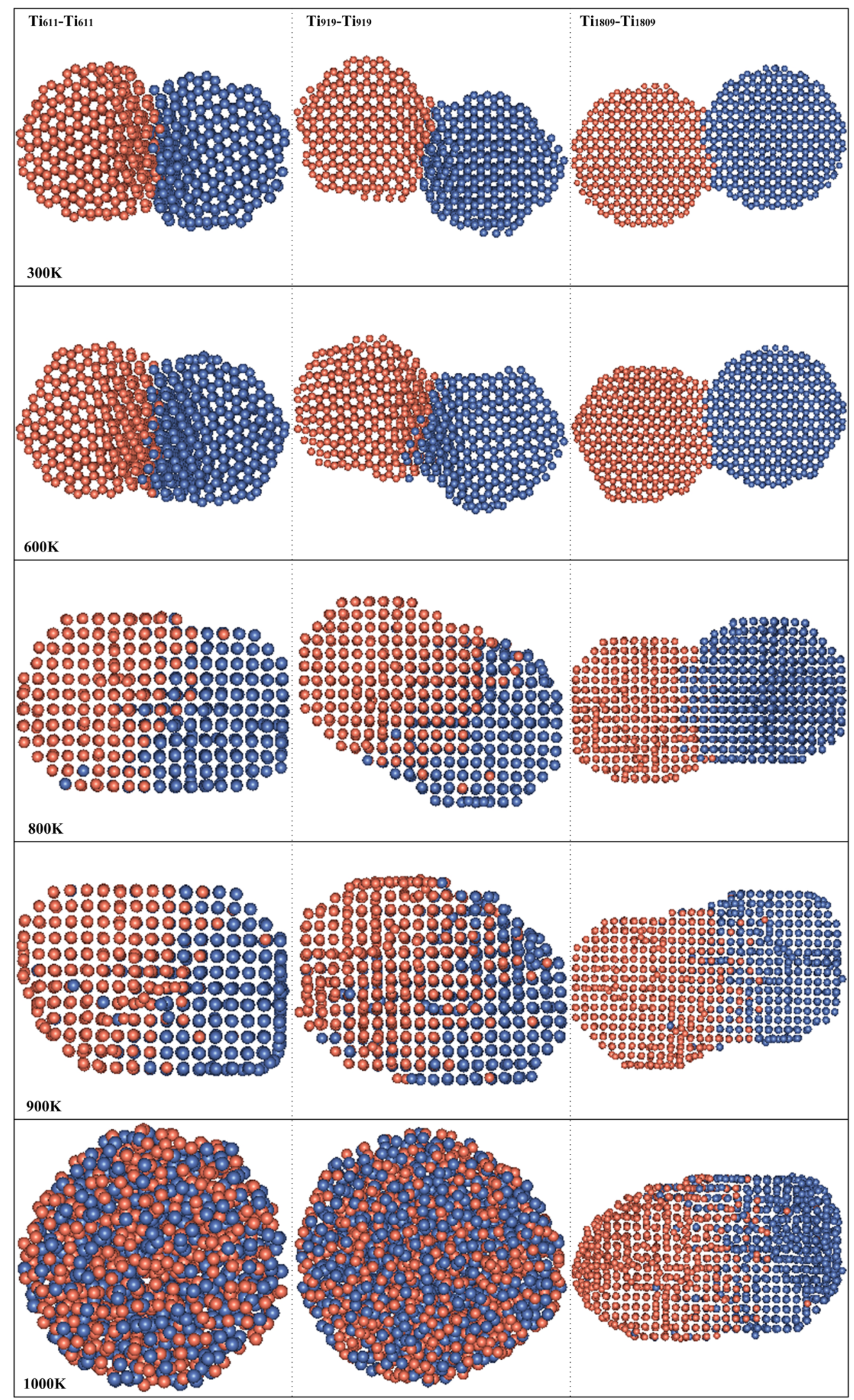

Fig. 6. Atomic packing of three pairs at different temperatures. 


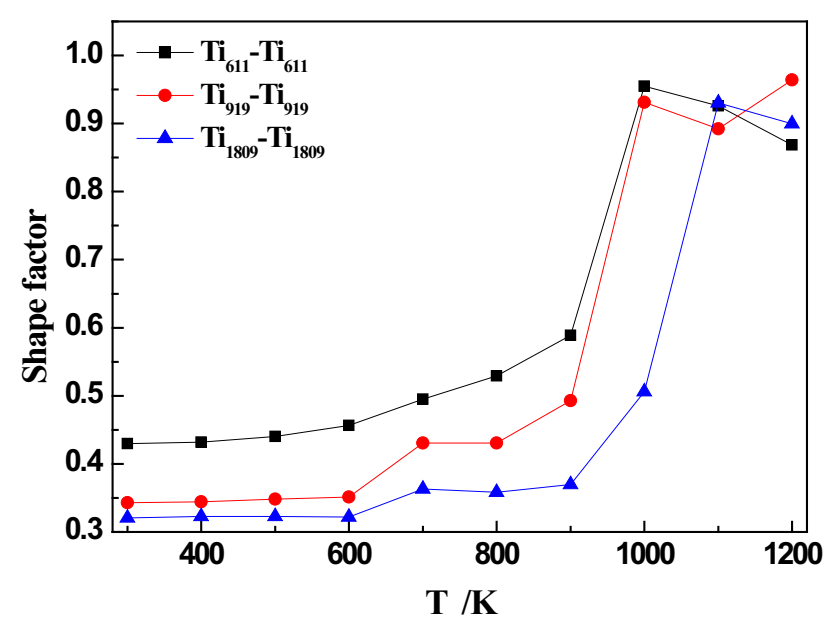

Fig. 7. Shape factors of three pairs, including $\mathrm{Ti}_{611}-\mathrm{Ti}_{611}, \mathrm{Ti}_{919}-$ $\mathrm{Ti}_{919}$, and $\mathrm{Ti}_{1809}-\mathrm{Ti}_{1809}$ as a function of temperature.

\section{ACKNOWLEDGEMENTS}

We acknowledge the financial support from the National Key R\&D Program of China (Grant No. 2016YFB0701304) and the National Natural Science Foundation of China (No. 51671051).

\section{REFERENCES}

1. S. Vergara, U. Santiago, C. Kumara, D. Alducin, R.L. Whetten, M.J. Yacaman, A. Dass, and A. Ponce, J. Phys. Chem. C 122, 26733 (2018).

2. C.E. Avila-Crisostomo, E. Sanchez-Mora, V. Garcia-Vazquez, and F. Pere-Rodriguez, J. Magn. Magn. Mater. 465, 252 (2018).

3. L.J. Lewis, P. Jensen, and J.L. Barrat, Phys. Rev. B 56, 2248 (1997).

4. J. Sopousek, O. Zobac, and J. Bursik, Phys. Chem. Chem. Phys. 17, 28277 (2015).

5. B. Buesser and S.E. Pratsinis, J. Phys. Chem. C 119, 10116 (2015).

6. Y. Ding, F.R. Fan, and Z.Q. Tian, Small 5, 2812 (2009).

7. F. Baletto and R. Ferrando, Rev. Mod. Phys. 77, 37 (2005).

8. C. Massobrio, A. Pasquarello, and A. Dal Corso, J. Chem. Phys. 109, 6626 (1998).

9. L. Zhang, Phys. Chem. Chem. Phys. 18, 7310 (2016).

10. R. Huang, Y.H. Wen, A.D. Voter, and D. Perez, Phys. Rev. Mater. 2, 126002 (2018).

11. P. Liu, M. Chandross, T.J. Boyle, B.G. Clark, and P. Vianco, APL Mater. 2, 022107 (2014).

12. N.E. Sharpless and R.A. DePinho, Nat. Rev. Mol. Cell Biol. 8, 703 (2007).

13. L. Zhang, Adv. Eng. Mater. 21, 1800531 (2019).

14. J. Stoltz, Regener. Med. Cell. Ther. 77, 111 (2012).

15. A.R. Amini, C.P. Laurencin, and S.P. Nukavarapu, Crit. Rev. Biomed. Eng. 40, 363 (2012).

16. B. Wysocki, J. Idaszek, K. Szlązak, K. Strzelczyk, T. Brynk, K.J. Kurzydlowski, and W. Święszkoski, Materials 9, 197215 (2016).

17. C.N. Elias, J.H.C. Lima, R. Valiev, and M.A. Meyers, JOM 60, 46 (2008).

18. H. Attat, S. Ehtemam-Haghighi, D. Kent, and M.S. Dargusch, Int. J. Mach. Tools Manuf. 133, 85 (2018).

19. A. Ataee, Y.C. Li, D. Fraser, G.S. Song, and C. Wen, Mater. Des. 137, 345 (2018).

20. A.T. Sidambe, Materials 7, 8168 (2014).

21. T.P.S. Sarao, H. Singh, and H. Singh, J. Therm. Spray Technol. 27, 1388 (2018).

22. S. Shaikh, S. Kedia, A.G. Majumdar, M. Subramanian, and S. Sinha, Appl. Phys. A Mater. Sci. Proc. 124, 821 (2018).
23. W.T. Huo, L.Z. Zhao, W. Zhang, J.W. Lu, Y.Q. Zhao, and Y.S. Zhang, Mater. Sci. Eng. C Mater. Biol. Appl. 92, 268 (2018).

24. K.Y. Cheng, N. Pagan, D. Bijukumar, M.T. Mathew, and M. McNallan, Thin Solid Films 665, 148 (2018).

25. V. Peretti, S. Ferraris, G. Gautier, C. Hellmich, O. Lahayne, B. Stella, S. Yamaguchi, and S. Spriano, Tribol. Int. 126, 21 (2018).

26. X.P. Li, J.V. Humbeeck, and J.P. Kruth, Mater. Des. 116, 352 (2017).

27. J.H. Jung, H.K. Park, B.S. Lee, J. Choi, B. Seo, H.K. Kim, G.H. Kim, and H.G. Kim, Surf. Coat. Technol. 324, 106 (2017).

28. A. Basalah, S. Esmaeili, and E. Toyserkani, J. Mater. Process. Technol. 238, 341 (2016).

29. Y.J. Liu, S.J. Li, H.L. Wang, W.T. Hou, Y.L. Hao, R. Yang, T.B. Sercombe, and L.C. Zhang, Acta Mater. 113, 56 (2016).

30. A. Rai, H. Helmer, and C. Körner, Addit. Manuf. 13, 124 (2017).

31. D. Bourell, J.P. Kruth, M. Leu, G. Levy, D. Rosen, A.M. Beese, and A. Clare, CIRP Ann. Manuf. Technol. 66, 659 (2017).

32. H.K. Park, Y.K. Ahn, B.S. Lee, H.H. Jung, C.W. Lee, and H.G. Kim, Mater. Lett. 187, 98 (2017).

33. L.C. Zhang and H. Attar, Adv. Eng. Mater. 18, 463 (2016).

34. D. Bourell, H. Marcus, J.W. Barlow, and J.J. Beaman, Int. J. Powder Metall. 28, 369 (1992).

35. H. Attar, M. Calin, L.C. Zhang, S. Scudino, and J. Eckert, Mater. Sci. Eng. A 593, 170 (2014).

36. V. Urlea and V. Brailovski, J. Mater. Process. Technol. 242 , 1 (2017).

37. B. Chen, J.H. Shen, X.X. Ye, J.K. Umeda, and K. Kondoh, J. Mater. Res. 92, 3769 (2017).

38. D. Herzog, V. Seyda, E. Wycisk, and C. Emmelmann, Acta Mater. 117, 371 (2016).

39. D.D. Gu, W. Meiners, K. Wissenbach, and R. Poprawe, Int. Mater. Rev. 57, 133 (2012).

40. W.J. Sames, F.A. List, S. Pannala, R.R. Dehoff, and S.S. Babu, Int. Mater. Rev. 61, 316 (2016).

41. L. Zhang, Sci. Adv. Mater. 9, 1768 (2017).

42. L. Zhang and Q.N. Fan, Indian J. Phys. 90, 9 (2016).

43. L. Zhang, J. Phys. Soc. Jpn. 85, 054602 (2016).

44. X. Qu, Mater. Sci. Technol. 33, 822 (2017).

45. B. Buesser and S.E. Pratsinis, J. Phys. Chem. 119, 10116 (2015).

46. A.M. Mazzone, Philos. Mag. B 80, 95 (2000).

47. I.V. Chepkasov, Y.Y. Gafner, and S.L. Gafner, J. Aerosol Sci. 91, 33 (2016).

48. A.L. Gould, A.J. Logsdail, and C.R. Catlow, J. Phys. Chem. 119, 23685 (2015).

49. C. Mottet, G. Rossi, F. Baletto, and R. Ferrando, Phys. Rev. Lett. 95, 035501 (2005).

50. L. Zhang and Q.N. Fan, J. Phys. Soc. Jpn. 82, 054601 (2013).

51. P. Grammatikopoulos and R.C. Pond, Acta Crystallogr. B 72, 67 (2016).

52. M.I. Mendelev, T.L. Underwood, and G.J. Ackland, J. Chem. Phys. 145, 154102 (2016).

53. L. Zhang, Prog. Nat. Sci. Mater. Int. 29, 237 (2019).

54. D. Farkas, Model. Simul. Mater. Sci. Eng. 2, 975 (1994).

55. R. Pasianot and E. Savino, Phys. Rev. B 12, 12704 (1992).

56. L. Lian, C.X. Su, and P.B. Armentrout, J. Chem. Phys. 97, 4084 (1992).

57. M. Sakurai, K. Watanabe, K. Sumiyama, and K. Suzuki, J. Chem. Phys. 111, 235 (1999).

58. P. Piseri, T. Mazza, G. Bongiorno, C. Lenardi, L. Ravagnan, F.D. Foglia, F. DiFonzo, M. Coreno, M. DeSimone, K.C. Prince, and P. Milani, New J. Phys. 8, 1 (2006).

59. P.L. Dulong and A.T. Petit, Ann. Chim. Phys. 10, 395 (1819).

Publisher's Note Springer Nature remains neutral with regard to jurisdictional claims in published maps and institutional affiliations. 